\title{
VISUAL SPATIAL INTERACTIONS BETWEEN DOTTED LINE SEGMENTS ${ }^{1}$
}

\author{
WILLLAM R. UTTAL \\ The University of Michigan, Ann Arbor, MI 48104, U.S.A.
}

(Received 30 June 1975; in revised form 1 October 1975)

\begin{abstract}
This study examines the interactions that occur between dotted line segments as a function of their separation and orientation using a dotted target detection paradigm. The results of the experiment indicate that it is the separation between interacting pairs of lines rather than shifts in retinal locus that account for the observed decline in detectability as the separation increases. However, factors of global organization or figural goodness seem also to exert a powerful influence on the detectability of the pattern. The results are interpreted in terms of a central, rather than a peripheral, model.
\end{abstract}

One of the most often enunciated generalizations of visual perception is that, compared to the presentation of a single part, multiple parts of a stimulus presented to different retinal loci interact in ways that can either enhance or reduce the perception of the whole stimulus. Many types of spatial interaction have been discovered, including reciprocal inhibition and spatial summation. Contour enhancement is usually attributed to the former (see Ratliff, 1965, for a summary), decreases in detection threshold, to the latter (see Kristofferson and Dember, 1958, for an example). The present study inquires into a form of spatial interaction that seems to fit into neither of these categories but is closer to the organizational interactions considered in the older Gestalt literature. In this paper, we are dealing with a form of spatial interaction that occurs over long distances and that is also sensitive to the cognitive quality, or figural goodness, of the pattern.

To explore this new form of spatial interaction, we determine the detectability of two colinear dotted-line segments as a function of their separation and orientation with respect to each other. The task requires that these target patterns be detected in a visual noise field composed of a number of randomly positioned dots-a paradigm that has proved to be quite powerful in assaying the perceptual effects of geometrysensitive pattern recognition tasks of this sort. A full description of the logic of this procedure is given by Uttal (1975).

The results of the present study indicate that, although an increase in separation between two dotted-line segments results in a decrease in detection when the two lines remain colinear, there is a more complex effect of separation when factors that we may call in the Gestalt tradition, "figural quality" are varied simultaneously. In the latter situation, some of the separation effects can be overridden, indicating that detection performance seems to be highly dependent on the global organization of the figure. This result has substantial implications for theories of pattern perception.

1 The research reported in this paper was supported by research grants from the National Institute of Mental Health (MH 24016) and by an NIMH Research Scientist Award (MH 29941-03) to the author.

\section{METHOD}

\section{Subjects}

Students at The University of Michigan served as Ss in the experiments reported in this paper. Each was paid a fixed stipend for a daily 1 hr session. No evidence of any visual abnormalities was reported, although some Ss wore corrective lenses. Prior to the collection of the experimental data, all $S$ s were extensively familiarized with the target stimuli. Unfortunately, different groups of Ss participated in each part of the experiment. Since individual differences are often relatively large, comparisons between absolute scores in different parts of the study are not meaningful.

\section{Apparatus}

Target stimuli were composed of dotted lines embedded within randomly positioned noise dots on the face of a cathode ray oscilloscope controlled by a small digital computer. The number of noise dots was constant for each daily session. A sample stimulus embedded in 20 noise dots is shown in Fig. 1. The computer supervised all stimulus presentations, response acquisition, and data analysis procedures. Target stimulus patterns were prepared in advance by the experimenter and then stored in the memory of the computer. A random number algorithm was used by the control program of the computer to counterbalance the number and order of the stimuli to be presented within each $S \mathrm{~s}$ hourly session. The pattern of random masking noise dots was calculated anew after each trial so that a novel mask was used in each trial



Fig. 1. A sample stimulus consisting of two three-dot horizontal segments embedded in a masking field of 20 random dots. 
The $S$ viewed the oscilloscope scteen from a distance of $33 \mathrm{~cm}$. The viewing region on the face of the oscilloscope on which the random noise dots were presented was $5.6^{\circ}$ by $5 \cdot 6^{2}$, but stimulus target patterns were smaller, typically subtending an angle of about $3^{\circ}$ by $3^{*}$. The luminance of each plotted dot was about $0.1 \mathrm{c}^{2} \mathrm{~m}^{2}$. Otherwise, the compartment in which the study was run was dark and sound-proofed.

Stimuli were plotted on the oscilloscope by presenting an intensification pulse that had a duration of $8 \mu \mathrm{sec}$ to the $Z$-axis input. However, the duration of each dot was determined more by the persistence of the P-15 phosphor of the oscilloscope's cathode ray tube than by this electronic pulse. According to the manulacturer's specifications the P.15 phosphor used in this instrument has a persistence such that the light output is reduced to 0.1 of $1^{\circ}$ of its initial brightness after $50 \mu \mathrm{sec}$. The apparent persistence of this briefly illuminated spot, of course, was even further elongated by the properties of the observer's visual system. The total physical duration of each stimulus, however, was defined by the number of masking and target dots involved and varied between 1 and $5 \mathrm{msec}$.

The $S$ 's head was constrained in a fxed viewing position by a forehead switch. Unless this forehead switch contact was closed, two hand-held response switches were inactive and no responses could be entered into the computer.

\section{Procedure}

The experimental procedure utilized a two-alternative forced-choice paradigm in which the $S$ was required to specify (by depressing one or the other of the two handheld switches) which of two sequentially presented stimuli (separated by $0.5 \mathrm{sec}$ ) contained a target pattern as well as the randomly positioned dotted visual noise common to both stimuli. The alternative (or nontarget) stimulus contained, in addition to the identical noise dot pattern, a number of randomly placed dots equal to the number in the target pattern in order to equate overall brightness and dot numerosity.

The set of targets that was presented in each experiment was thoroughly familiar to the $S$. In each case he knew which targets set was to be used on any given day. Each set was designed to introduce uncertainty about locus. orientation and arrangement as shown in Figs. 2, 3 and 4.

Following the depression of either one of the response switches, the $S$ was informed of the correctness or incorrectness of his response to the previous trial by means of a plus or a minus sign plotted on the oscilloscope screen.

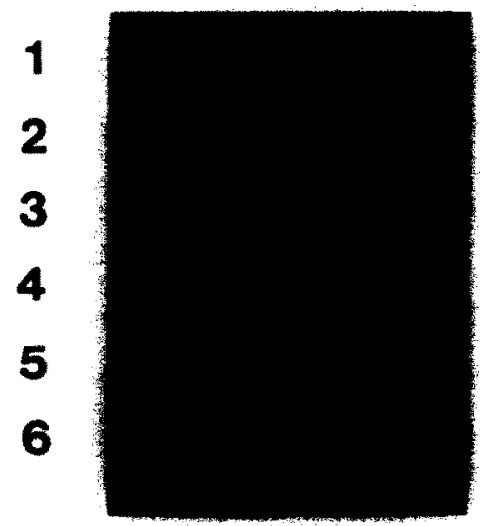

Fig. 2. Samples of the stimuli used in experiment 1. In addition to this horizontal set, another similar set of vertical lines was also used. Separation between the two threedot line segment pairs is successively $0.6^{\circ}, 0.9^{\circ}, 1.25^{\circ}, 1.70^{\circ}$ and $2 \cdot 15^{\circ}$ of visual angle. Only one of these stimuli appeared in each trial.

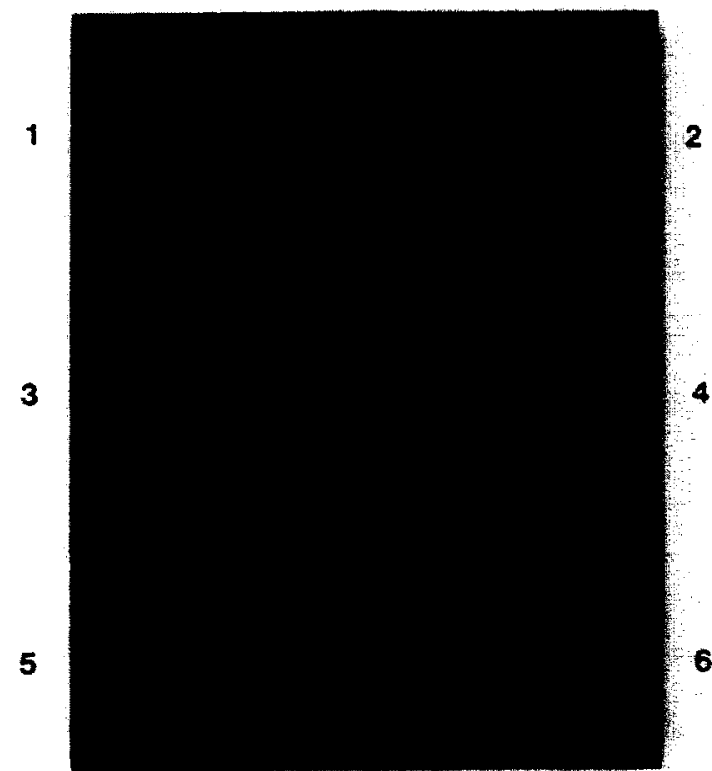

Fig. 3. Samples of the stimuli used in experiment 2 . The corners have been added to the photographs to emphasize the location of the stimulus lines but did not appear in the experimental trials. Each of these figures exemplifies a particular position and separation. All five separations were presented at all four positions, as was the single threedot line segment, for at total of 24 different stimuli.

This feedback signal, which lasted for $0.5 \mathrm{sec}$, also served as a fixation cue; no other fixation cue, except the stimulus itself, was present in the intertrial or interstimulus intervals. The depression of the response switch and the presentation of the feedback signal was followed automatically

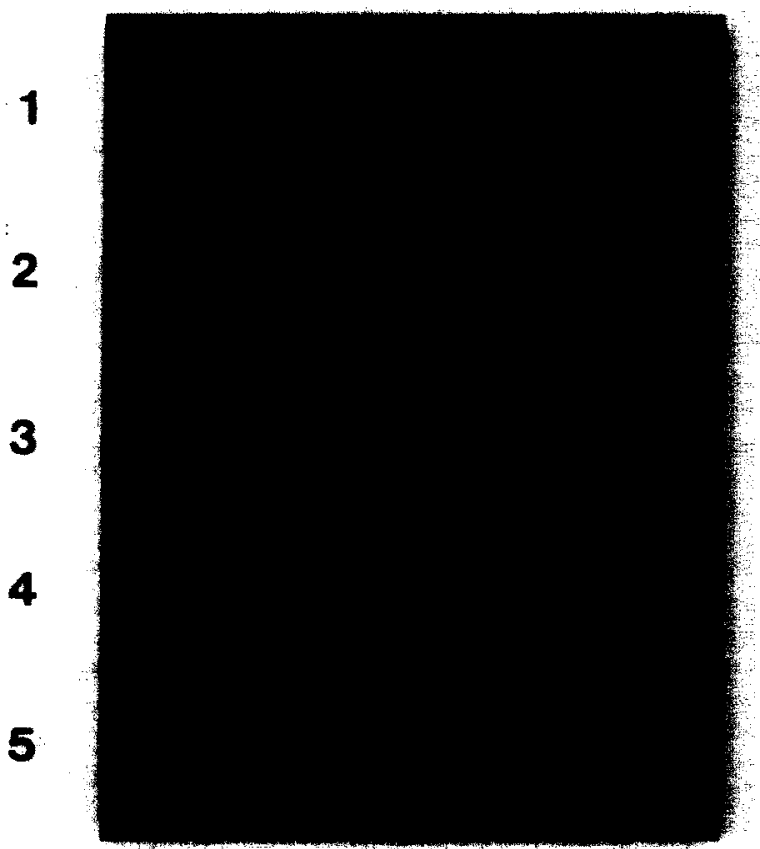

Fig. 4. Samples of the stimuli used in experiment 3 . In addition to this horizontal set, another equivalent set of vertically oriented patterns was also used. The separation between the ends of the two three-dot segments was always $1.2^{2}$ of visual angle. The angle of rotation between the axis of the two lines could be $0^{\circ}, 22.5^{\circ}, 45^{\circ}, 67.5^{\circ}$ and $90^{\circ}$. respectively. 
by the presentation of the two stimuli of the next trial Pacing, therefore, was a function of the rate at which the $S$ responded.

All performance scores were measured as the percentage of the total number of presentations for which the target was correctly detected. A score of $50^{\circ}$. represents chance performance in this two-alternative task.

Data was accumulated for each $S$ and punched on a paper tape at the end of each session. At the end of the day, a secondary data analysis program was used to summarize the results from all $S$ s.

The results of the three experiments are plotted on each of their respective graphs as a function of the major geometric independent variable manipulated in each experiment and parametrically as a function of the noise dot density. Noise dot density is scanned in order to make explicit any differential effects of the signal-to-noise ratios. Although no particularly strong bottom or top effects occurred in this study, the results of some similar experiments had been profoundly affected by variations of noise dot density. In order to emphasize the general effects of each variable, the data from all noise dot density curves are averaged for each experiment to produce an "average value curve." The average value curves for all three experiments are plotted together in Fig. 8.

\section{Experiment 1}

Experiment 1 is the prototypical experiment of this study. Its original purpose was to determine the effect of distance between two three-dot line segments. Two sets of six stimuli, one oriented vertically and one oriented horizontally, were used. The horizontal set of these target stimuli is shown in Fig. 2. Each six-dot target in each set was designed with progressively increasing distance between the two three-dot line segments. Each dot in each of the three-dot line segments was separated from its neigh bors by $36^{\prime}$ of visual angle. The separation between the two line segments was progressively $0.6^{\circ}, 0.9^{\circ}, 1.25^{\circ}, 1.70^{\circ}$ and $2 \cdot 15^{\circ}$. Note that, in addition to the type of two-line segment stimuli shown in Fig. 2 , there is also one additional target stimulus consisting of a single three-dot line segment. This three-dot target stimulus was presented to provide a reference against which the performance for the other two-line targets could be compared to determine if there was any separation at which the pairs of three-dot lines were no longer interacting.

On 8 successive days, the stimuli were presented in increasing amounts of dotted visual noise. Noise dot numerosities of $30,40,50,60,70,80,90$ and 100 dots were used. On 8 succeeding days, the same noise dot densities were used in reverse order. The data from the 8 pairs of days with corresponding noise dot numerosities were then pooled to produce the final data, which was thus balanced for any quotidian sequence effect.

Since each stimulus was presented in each condition approx 150 times to each of four $S$ s during two equivalent sessions, each data point in Fig. 5 represents the sample of approx 600 trials.

\section{Experiment 2}

Although experiment 1 was designed to measure the effects of intersegment distance, it should be explicitly noted that there are, in fact, two dimensions varying simultaneously that could account for the obtained results. First, as specified, the separation between the two line segments is varying. Second since the interdot spacing in each line is held constant the retinal locus of each line segment is progressively more peripheral. Thus experiment 1 is confounded in this regard, and it is not possible to specify whether retinal locus or segment separation is the fundamental cause of the obtained result.

A number of different alterations in the stimulus targets were considered to resolve this confounding. Attempts

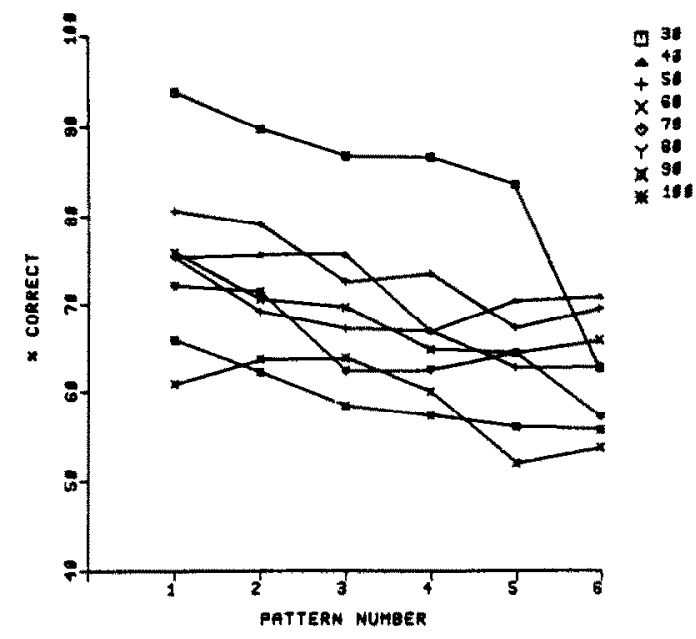

Fig. 5. The results of experiment 1. Refer to the text to understand why these data are not meaningful except in comparison with those of experiment 2 . The family of curves is parametric with the number of dots in the mask-

ing field. Pattern numbers are as indicated in Fig. 2.

were made to keep the stimulus extent constant by progressively reducing the interdot distance in each line segment as the intersegment separation increased. Since dot density also exerts a powerful, if not the most powerful, effect on dotted line detection (Uttal, 1975). this simply shifted the confounding from one kind to another and, worse, the resulting data were so complicated as to be essentially meaningless.

The alternative stimulus chosen to overcome this confounding was not entirely satisfactory either, but it represented a considerable improvement over the original set. It, too. is still susceptible to the sort of confounding that confused experiment 1 , although to a much lesser degree. Six of the set of 24 stimuli used in experiment 2 are shown in Fig. 3.

The expedient in experiment 2 was to place the pair of dotted line segments at any one of four peripheral edges of the square visual field. All of these target stimuli were placed $1.8^{\circ}$ peripheral from the fixation point. In this manner, as the separation between the two dotted-line segments was increased, each of the segments moved only to a slightly more peripheral location. Furthermore, the stimuli were outside the central retinal region at which the largest magnitude changes in acuity and locus sensitivity effects would be expected to occur. Thus the slight shift in peripheral locus should have minimized, if not eliminated, any residual locus effect. Then. if the results of the spacing shift were the same for experiments 1 and 2. the effect observed in experiment 1 may be mainly attributed to intersegment spacing, rather than the confounding variable, locus.

The target stimuli used in experiment 2 were the same in all respects, except retinal locus, as those used in experiment 1. The same sequence of experimental sessions (eight in ascending order of noise density and eight in descending order) was also used, as in experiment 1 . Since each target stimulus was presented in each condition approx 250 times to each of four $S s$ on two equivalent sessions, each data point on Fig. 6 represents the sample of approx 1000 trials.

\section{Experiment 3}

Experiment 3 was designed to determine the effects of a change in performance using target stimuli in which the separation between the ends of two line segments remained constant, but in which the pattern or global geometry of the figure varied. Five samples of the target stimuli used in this experiment are shown in Fig. 4. A set of vertically 


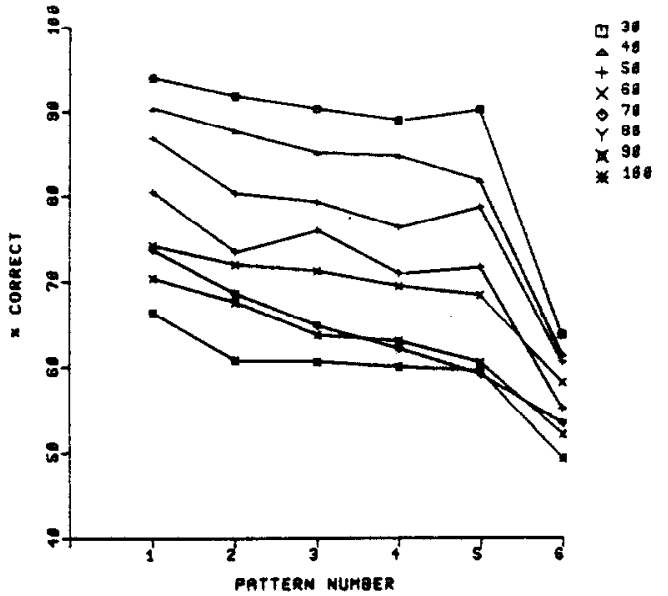

Fig. 6. The results of experiment 2. There is a gradual decline in detectability as the separation increases, a sharp decline when only a single three-dot line segment is presented. The family of curves is parametric with the number of dots in the masking field. Pattern numbers are as indicated in Fig. 3.

oriented stimuli, similar to those in Fig. 4, was also used. Since it is known that there is no oblique effect with dotted patterns (Uttal, 1975), there was no need to control for this possible artifact in this experiment. For all members of this set of stimuli, one dotted-line segment remains permanently fixed, while the other segment rotates, maintaining a constant distance $\left(1.2^{3}\right)$ between the end dots of the two segments. The angles of rotation that could be assumed by the stimuli were $0^{\circ}, 22 \cdot 5^{\circ}, 45^{\circ}, 67 \cdot 5^{\circ}$, and $90^{\circ}$. Note that the effect of this rotation is actually to decrease the actual average physical distance between the constituent dots of the two line segments as the angle of rotation increases. As we shall see, however, an even more important variable is that the global pattern also changes; the target stimulus varies from a straight line through increasingly acute angles, to a right-angle pattern. In other words, it goes from one good figure through some poorer organizations to another good figure. In this way, a test is made of the effects of simultaneous variation of simple geometri-

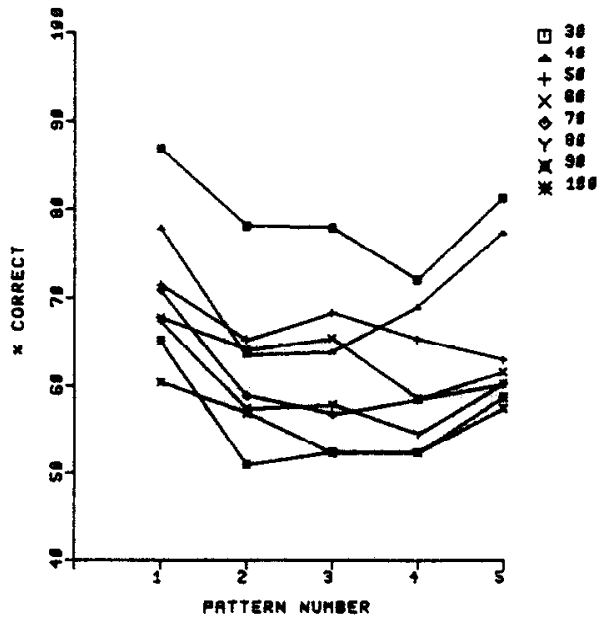

Fig. 7. The results of experiment 3. Note the U-shaped curve that is obtained because of the relatively high detectability of straight lines and the right angle. The family of curves is parametric with the number of dots in the masking field. Pattern numbers are as indicated in Fig. 4. cal propinquity, on the one hand. and figural quality, or goodness. on the other.

The same sequence of experimental trials and the same noise dot densities were used in experiment 3 as in the preceding two experiments. Since each stimulus was presented 150 times to each of four Ss, each dot on Fig. 7 represents a sample of approx 600 trials.

\section{RESL LTS}

\section{Experiment 1}

The results of experiment 1 are shown in Fig. 5 . In this confounded situation. in which both the retinal locus of and the separation between the two line segments are simultaneously changing, there is a gradual decline in the detectability of the two three-dot line segments for all levels of noise. Due to the confounding of this experiment, already described. this result can only tell us that the combined effect of both a shift to the periphery and an increase in line segment separation is to reduce the detectability of the target stimuli.

As the intersegment separation increased, the final level to which the performance scores declined appeared to be very close to the values obtained for a single three-dot line segment. This result, however, is also equivocal, for the three-dot line segment was placed at the fixation point, while the pair of line segments were considerably more peripheral. It is possible that the central locus might have artificially enhanced the score for the single three-dot line segment. in the same way that retinal locus might explain the major result of experiment 2 . All in all, this initial experiment provides little that can be used to resolve the question of whether it is the separation or the locus change that produces the effects observed when dotted line segments are moved apart. Nevertheless, experiment 1 is necessary; for, just as an algebraic problem with two unknowns requires two equations to determine the solution, it is only by comparing the results of experiments 1 and 2 that we can determine whether it is separation or retinal locus that produces the variation in performance.

\section{Experiment 2}

The results of experiment 2 are shown in Fig. 6 . In this experiment, by placing the two dotted-line segments at the periphery of the noise field, only minimal changes in retinal locus are introduced as the separation between the two dotted-line segments is varied over its full range. The magnitude of the resulting effect (in this case, of the variation of the separation between two dotted-line segments) is seen in Fig. 6 to be nearly equivalent to that of experiment 1 . It is only when a target stimulus consisting of only a single three-dot line segment is used that there is any substantial reduction in the performance of the Ss. This indicates that, in fact, the performance for two three-dot line segments does not decline to the level of performance for a single three-dot segment, as indicated in experiment 1 . As suspected, the similarity of the scores for two widely separated three-dot segments and a single three-dot segment in experiment 1 is probably an artifact of the locus, and the two segments are still interacting or displaying some simple sort of probability summation at the separations used. 


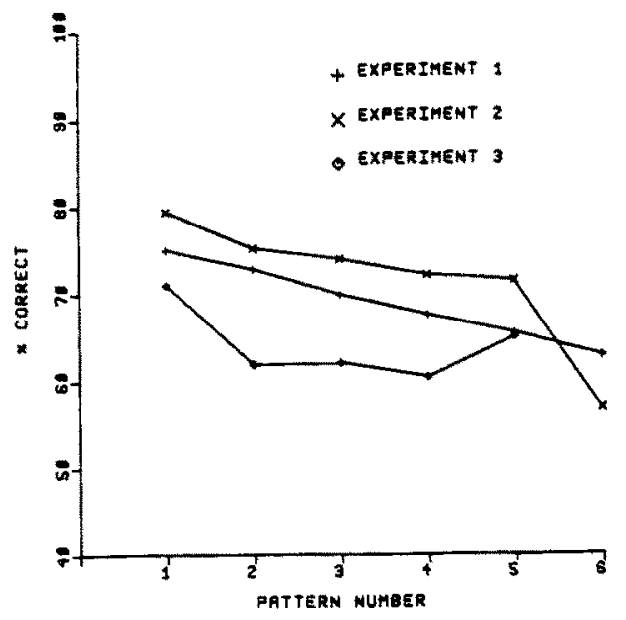

Fig. 8. The average value curves for the three experiments. obtained by simply averaging all values for each pattern at all noise levels.

\section{Experiment 3}

The results of experiment 3 are shown in Fig. 7 . As the variable line segment rotates about the fixed line to its first nonlinear condition $\left(22.5^{\circ}\right)$, there is an immediate decrease in the detectability of the combined stimulus. The detectability scores then remain nearly constant for the next two conditions of rotation $\left(45^{\circ}\right.$ and $67.5^{\circ}$, respectively). However, when the stimulus line segments are oriented at right angles to each other, there is a marked increase in detectability scores. It should be remembered that throughour this entire process, there has been a gradual decrease in the overall spacing between the components of the two line segment dots as the angle of rotation increased. Yet, once linearity is violated to any degree, little further change in the detection score occurred until another regular pattern-a right angle-was attained.

\section{General}

The results of all three experiments demonstrate the well-established signal-to-noise ratio effect known from many of our previous experiments. As the noise dot density increases, there is a progressive decrease in the $\mathrm{Ss}$ ' detection performance.

However, more important, in the context of the present study, is the relative effect within each noise dot density condition in each experiment. Figure 8 plots the average values of each of the three experiments. Obviously, the difference between the relative effects of experiments 1 and 2 is very small. The maximum score difference of $9.6 \%$ in experiment 1 occurs for the conditions in which the two line segments were separated by only $36^{\circ}$, on the one hand, and $2.15^{\circ}$, on the other. The comparable difference for experiment 2 is $8 \%$.

Though it is interesting to note that the absolute values of the scores for corresponding conditions in experiment 2 are actually higher than the scores for experiment 1 (despite the fact that the target patterns in experiment 2 were more peripheral), this effect is probably due to the fact that different groups of $S_{s}$ with somewhat higher average scores were used in experiment 2. On the other hand, the similarity of the absolute levels of the two sets of data does suggest that retinal locus plays only a small role in defining the detectability of these dotted target patterns and that most of the effect of increased separation must be attributed to a lessening of the interaction between the two line segments.

An equally compelling conclusion from the average value curve of experiment 3 is that effects of figural goodness can overwhelm the effects of simple geometrical distance.

\section{DISCUSSION}

The overall goal of research of the kind exemplified by the experiments of this present study is to develop a psychophysics of form; that is, to develop an understanding of the relative contribution of a number of organizational variables to the perception of form. The obstacles to achieving this goal in the past have resulted from the twin facts that neither are the dimensions of form easy to quantify nor was an assay procedure a vailable that could deal with only a single aspect of the complex of processes that we call visual perception. The dot masking procedure provides the assay technique, and the results of these experiments, if not the contrived stimulus patterns themselves, are steps toward the quantification of form.

For the particular types of stimuli used in this study, the general results indicate that, in addition to the expected effects of geometrical propinquity, there are factors operating that involve complexity and figural goodness, dimensions that are both diffcult to quantify. (In our terms, a right angle is a better form than a more obtuse angle.)

The specific purpose of this study was to determine why increases in the separation between two dottedline segments led to a progressive decline in the detectability of the combined stimulus. The problem was originally confounded in experiment 1 by the fact that both segment separation and retinal locus must simultaneously vary in this experimental design. In experiments 2 and 3 , alternative experimental designs were established that partially controlled this confounding. Experiment 2, through placing the two line segments at the periphery, produced results that, when compared with the findings of experiment 1. suggested that the retinal locus of the two line segments played only a secondary role in the variation of detectability. Similarly, in experiment 3, even though the physical spacing of the two line segments was actually continuously decreasing as the angle between the two lines varied, the results indicated that a factor of a variable we may call figural goodness played a more important role. Performance was highest only when good figures - a straight line or a right angle-were the targets.

Thus the results of experiment 2 suggest that the actual physical separation between the two segments of the dotted line is a more important variable than the retinal locus effect. Experiment 3, however, says that an even more important dimension than physical separation is figural goodness. We thus have established an ordering of relative effectiveness for the infuence of these three variables: figural goodness, physical separation and retinal location. 
It is apparent from these results that dot detection tasks deal with a hierarchy of different kinds of interactions. At one level are the interactions that occur between dots in a single line or curve. At another level are the more global interactions that occur between multiple lines and curves that collectively define a more complex figure.

What kind of mechanism could be selectively sensitive to these and related dimensions and exhibit the same sort of hierarchy of interactions? One possible mechanism is a two-dimensional sheet of neurons that collectively evaluates an autocorrelation-type algorithm. An extensive discussion of such a distributed nerve net model and data on other forms of polygonal stimuli has already appeared (Uttal, 1975).

Though it would be inappropriate to repeat the argument at this point, it is interesting to note that the type of distributed nerve network that is sensitive to the global dimensions of form and shape may also be considered to be a model of a central, rather than a peripheral, nervous structure. Similarly. the high sensitivity of the task in the present study to goodness of form suggests that many uspects of dor detection are mediated by complex central nerrous functions (some would say cognitive processes) rather than by simple geometrical lateral interactions in the periphery. Therefore, the search for simple neural mechanisms sensitive to propinquity alone would be futile in any attempt to explain these results. Human observers seem, in the context of this study, to be more concerned with the global organizational properties of a figure than with its local geometrical features.

\section{REFEREYCES}

Kristofferson A. B. and Dember W. N. (1958) Detectability of targets consisting of multiple small points of light. Rept of Project Michigan (No. 2144-298-T), Willow Run Laboratories. Univ. of Michigan. Sept. $19 \div 8$.

Ratliff F. (1965) Wach Bands: Quantitatice Studies on Neural Networks in the Retina. Holden-Day. San Francisco.

Uttal W. R. (1975) An Autocorrelation Tikory of Form Detection. Lawrence Erlbaum. Hillsdale. $\mathrm{V}$. 